\title{
Lunar laser ranging test of the Nordtvedt parameter and a possible variation in the gravitational constant
}

\author{
F. Hofmann ${ }^{1,2}$, J. Müller ${ }^{1,2}$, and L. Biskupek ${ }^{1}$ \\ 1 Institut für Erdmessung (IfE), Leibniz Universität Hannover, Scheiderberg 50, 30167 Hannover, Germany \\ e-mail: hofmann@ife.uni-hannover.de \\ ${ }^{2}$ QUEST - Centre for Quantum Engineering and Space Time Research, Leibniz Universität Hannover, Welfengarten 1, \\ 30167 Hannover, Germany
}

Received 30 August 2010 / Accepted 19 October 2010

\section{ABSTRACT}

\begin{abstract}
Context. Forty years of lunar laser ranging (LLR) data provide an excellent basis to determine various parameters of the Earth-Moon system as well as parameters related to gravitational physics.

Aims. We update the Institut für Erdmessung (IfE) LLR model taking the effect of a fluid lunar core into consideration. The temporal variation in the gravitational constant $\dot{G} / G_{0}$ and the strong equivalence principle, parameterized by the Nordtvedt parameter $\eta$, are investigated.

Methods. A set of LLR observations from 1970 to 2009 was analysed and the parameters were determined by a least squares adjustment in two runs. After solving for classical Newtonian parameters (e.g. initial conditions for the lunar orbit and rotation) in the first run, relativistic parameters were determined in the second run.

Results. The upper limits to the gravitational constant and the Nordtvedt parameter were found to be $\dot{G} / G_{0}=(-0.7 \pm 3.8) \times 10^{-13} \mathrm{yr}^{-1}$ and $\eta=(-0.6 \pm 5.2) \times 10^{-4}$.
\end{abstract}

Key words. Moon - gravitation - celestial mechanics - ephemerides

\section{Introduction}

Since the first passive retroreflector on the lunar surface was deployed by the Apollo 11 mission, measurements of round trip travel times of laser pulses between stations on the Earth and retroreflectors on the Moon have been carried out for over 40 years. The weak signal-to-noise ratio of the pulses is mainly caused by atmospheric beam divergence and corner cube diffraction, and makes lunar laser ranging (LLR) challenging. Therefore, only a few observatories worldwide are capable of tracking the Moon. The measured lunar returns over a period of 15 to 20 min are combined to so-called normal points (NP). Up to now, approximately 17000 NPs were collected and serve as observations for the analysis. Various components of the EarthMoon system dynamics can be addressed, e.g., initial conditions for the lunar orbit and rotation, reflector and station coordinates, Earth orientation parameters (Biskupek \& Müller 2009; Zerhouni \& Capitaine 2009), nutation coefficients (Biskupek et al. 2009), and also studies of the lunar interior (Williams \& Boggs 2009). In addition, LLR is used to test general relativity (Müller et al. 2008), e.g., the strong equivalence principle (EP) (Müller \& Nordtvedt 1998; Williams et al. 2009; Nordtvedt et al. 1995 ), temporal variation in the gravitational constant $G$ (Müller \& Biskupek 2007; Williams \& Folkner 2009), metric parameters, and preferred frame effects (Soffel et al. 2008). Here, we focus on the strong equivalence principle and a possible temporal variability of the gravitational constant after refining the analysis model for the lunar rotation taking into account the effect of a fluid core and an updated data series (March 1970 to December 2009). The knowledge of more tighter limits of these quantities may be helpful to verify Einstein's theory or to distinguish between cosmological models and alternative theories of gravitation. Some theories predict for instance an EP violation at a level of $m_{\mathrm{g}} / m_{\mathrm{i}}=10^{-18}-10^{-12}$ with gravitational mass $m_{\mathrm{g}}$ and inertial mass $m_{\mathrm{i}}$ (Damour et al. 2002; Turyshev 2008), while some multidimensional theories expect a temporal variation in $G$ of the order of $\dot{G} / G=10^{-14}-10^{-11} \mathrm{yr}^{-1}$ (Steinhardt \& Wesley 2010; Sanders et al. 2010).

This Letter is organized as follows. Section 2 describes the analysis model and Sect. 3 shows the basis of the parameter estimation with a special focus on the investigated relativistic parameters. The results obtained are discussed in Sect. 4 and our conclusions are given in Sect. 5.

\section{Analysis model}

The analysis model used at Institut für Erdmessung (IfE) is based on Einstein's theory of gravity. It is fully relativistic and complete up to the first post-Newtonian $\left(1 / c^{2}\right)$ level (see Müller et al. 2008). The simplified observation equation for the stationreflector distance $d$ (see Fig. 1) is

$d=c \frac{\tau}{2}=\left|\boldsymbol{r}_{\mathrm{EM}}-\boldsymbol{r}_{\mathrm{obs}}+\boldsymbol{r}_{\mathrm{ref}}\right|+c \Delta \tau$,

with the speed of light $c$, pulse travel time $\tau$, vector between geocenter and selenocenter $\boldsymbol{r}_{\mathrm{EM}}$, the position vector of the observatories $\boldsymbol{r}_{\mathrm{obs}}$, the position vector of the reflectors $\boldsymbol{r}_{\text {ref }}$, and a delay $\Delta \tau$ due to atmospheric and relativistic effects. To apply Eq. (1), all vectors must be given in the same inertial reference 


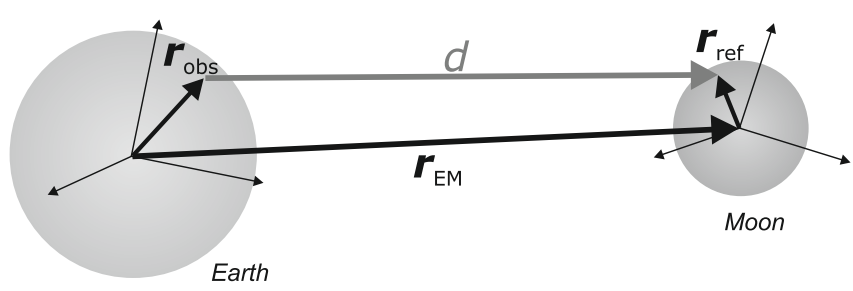

Fig. 1. LLR measurement setup with basis vectors.

frame. The vectors $\boldsymbol{r}_{\mathrm{obs}}$ and $\boldsymbol{r}_{\text {ref }}$ are transformed from their original terrestrial (TRF) or selenocentric (SRF) reference frame by applying

$$
\begin{aligned}
\boldsymbol{r}_{\mathrm{obs}} & =\boldsymbol{R}^{\mathrm{e}}(t) \boldsymbol{r}_{\mathrm{obs}}^{\mathrm{TRF}}, \\
\boldsymbol{r}_{\mathrm{ref}} & =\boldsymbol{R}^{\mathrm{m}}(t) \boldsymbol{r}_{\mathrm{ref}}^{\mathrm{SRF}} .
\end{aligned}
$$

The rotation matrix between TRF and inertial frame $\boldsymbol{R}^{\mathrm{e}}(t)$ is calculated from the Earth orientation parameters as described in McCarthy \& Petit (2004). For the rotation $\boldsymbol{R}^{\mathrm{m}}(t)$ between SRF and inertial frame, the lunar rotation vector $\omega^{\mathrm{m}}$ is required, which is obtained by numerical integration of the EulerLiouville equations for the Moon. The previous model for an elastic, homogeneous, and dissipative Moon has been extended by including the influence of a liquid core (Williams \& Boggs 2009). Adopting the expressions of Hinderer et al. (1982) for the Moon, this leads to Eq. (4) for the whole Moon, Eq. (5) for the core, and Eq. (6) for the coupling torque at the core mantle boundary $\boldsymbol{T}_{\mathrm{cmb}}$

$$
\begin{aligned}
\frac{\mathrm{d}\left(\boldsymbol{C}^{\mathrm{m}} \boldsymbol{\omega}^{\mathrm{m}}+\boldsymbol{l}^{\mathrm{c}}\right)}{\mathrm{d} t}+\omega^{\mathrm{m}} \times\left(\boldsymbol{C}^{\mathrm{m}} \boldsymbol{\omega}^{\mathrm{m}}+\boldsymbol{l}^{\mathrm{c}}\right) & =\boldsymbol{T}_{\mathrm{G}}, \\
\frac{\mathrm{d}\left(\boldsymbol{C}^{\mathrm{c}} \boldsymbol{\omega}^{\mathrm{c}}\right)}{\mathrm{d} t}+\omega^{\mathrm{m}} \times \boldsymbol{C}^{\mathrm{c}} \boldsymbol{\omega}^{\mathrm{c}} & =-\boldsymbol{T}_{\mathrm{cmb}}, \\
\boldsymbol{T}_{\mathrm{cmb}} & =K\left(\omega^{\mathrm{c}}-\omega^{\mathrm{m}}\right),
\end{aligned}
$$

with the additional angular momentum $\boldsymbol{l}^{\mathrm{c}}$ due to different core rotation

$l^{\mathrm{c}}=C^{\mathrm{c}}\left(\omega^{\mathrm{c}}-\omega^{\mathrm{m}}\right)$,

$\mathrm{m}$ and $\mathrm{c}$ superscripts indicate the whole Moon and the lunar core, respectively, $\boldsymbol{C}$ is the corresponding tensor of inertia, $\omega$ the rotation vector, $\boldsymbol{T}_{\mathrm{G}}$ the external gravitational torque, and $K$ a coupling parameter. Initial values for $\omega^{\mathrm{c}}$ used to integrate Eq. (5) as well as values for $\boldsymbol{C}^{\mathrm{c}}$ and $K$ are taken from Williams et al. (2001) and Williams \& Boggs (2009). These values are kept fixed in our solution and have not been estimated. The Earth-Moon vector $\boldsymbol{r}_{\mathrm{EM}}$ in Eq. (1) is calculated by numerical integration of the relativistic Einstein-Infeld-Hoffmann equations of motion with additional Newtonian accelerations due to the gravitation of the solar system bodies as well as gravity field inhomogeneities.

The inclusion of the lunar core refines the modelling of the lunar rotation and leads to an improved LLR analysis model. The root mean square of the post-fit residuals of the whole data series yields a reduction of $4 \mathrm{~mm}$ or $1.1 \%$ with the core extension.

\section{Parameter estimation}

The model is adjusted to the observations by performing a weighted least squares fit with two parameter groups. The first group are the non-relativistic Newtonian parameters, which are estimated in a first run of the analysis, with fixed relativistic parameters to their Einsteinian values, until a stable solution is obtained. These parameters include coordinates of LLR observatories and retroreflectors, $G M_{\mathrm{E}+\mathrm{M}}$, long periodic nutation coefficients, initial conditions for the lunar orbit and rotation, the lowest mass multipole moments of the Moon, lunar Love number, as well as a dissipation parameter and a lag angle indicating the lunar tidal acceleration. After convergence is achieved, single relativistic parameters are estimated together with the Newtonian parameters. All other relativistic parameters are fixed to their Einsteinian values. Tests relating to the gravitational constant and the EP are discussed in more detail below.

\subsection{Gravitational constant}

The gravitational constant $G$ entering into the equations of motion is described as

$G=G_{0}\left(1+\frac{\dot{G}}{G_{0}} \Delta t\right)$,

where $\Delta t=t-t_{0}$ is the time difference to the reference epoch $t_{0}=\mathrm{J} 2000$ and $\dot{G} / G_{0}$ as linear trend of the gravitational constant. This yields $\dot{G} / G_{0}=(-0.9 \pm 4.0) \times 10^{-13} \mathrm{yr}^{-1}$ when only the updated data series is considered and $\dot{G} / G_{0}=(-0.7 \pm 3.8) \times$ $10^{-13} \mathrm{yr}^{-1}$ including the core extension. Owing to modelling inadequacies and remaining systematic errors, the formal error from the fit was scaled by a factor of 2 to get a more realistic uncertainty. The scaling factor was inferred by different tests from the determined uncertainty in $\dot{G} / G_{0}$, e.g., by varying values of some fixed Newtonian parameter, such as the Earth-Moon orbit around the Sun, or the weighting of the individual measurements. For a comprehensive error analysis, we refer to Müller \& Biskupek (2007).

\subsection{Nordtvedt parameter}

Compared to laboratory masses, Earth and Moon have a large amount of gravitational self-energy. Therefore, they can be used as bodies for testing the strong EP with respect to the gravitational field of the Sun (Nordtvedt 1968a,b). If the EP is violated (Nordtvedt parameter $\eta \neq 0$ ), the Earth and the Moon will be accelerated differently by the Sun, resulting in a shifted lunar orbit in the solar direction. In the IfE LLR analysis program, this can be handled by determining the difference of the ratio of gravitational to inertial mass $\Delta\left(m_{\mathrm{g}} / m_{\mathrm{i}}\right)$ between the Earth and Moon in the equations of motion or by synodic Earth-Moon range oscillations towards the sun, parameterized by $\eta$, which leads to an equivalent result (Müller \& Nordtvedt 1998). In the present study, only the second procedure with the range term $\Delta r_{\mathrm{EM}}$ was considered

$\Delta r_{\mathrm{EM}}=A \eta \cos D$

where $D$ is the synodic angle and the amplitude of $A=12.8 \mathrm{~m}$ is derived from theoretical calculations by Nordtvedt (1995). This yields $\eta=(2.1 \pm 5.3) \times 10^{-4}$ considering only the updated data series and $\eta=(-0.6 \pm 5.2) \times 10^{-4}$ with the core extension. When using the realistic uncertainty value of $\eta=5.2 \times 10^{-4}$, Eq. (9) would lead to a maximum additional range of $7 \mathrm{~mm}$ along the Earth-Sun direction during the new and full Moon phases. For the mass ratio $\Delta\left(m_{\mathrm{g}} / m_{\mathrm{i}}\right)$, this yields $\Delta\left(m_{\mathrm{g}} / m_{\mathrm{i}}\right)=$ $-4.45 \times 10^{-10} \eta=0.3 \pm 2.3 \times 10^{-13}$, where we refer to Williams et al. (2009) for more details and Müller \& Nordtvedt (1998) for a dedicated error analysis: this procedure is carried out in a similar way to that indicated in Sect. 3.1. 


\section{Discussion}

We have succeeded in reducing the uncertainty in the current variation in $G$ by a factor of 2 , compared to previous IfE results (Müller et al. 2008). This is mainly due to the longer data series used and to a smaller extent a result of the refined modelling. The analysis shows a high correlation of $\dot{G} / G_{0}$ with the lag angle, which indicates the secular tidal acceleration. At the moment, this prevents a more precise determination of $\dot{G} / G_{0}$. Together with Williams \& Folkner (2009), this paper gives the actual highest accuracy of $\dot{G} / G_{0} \approx 4 \times 10^{-13} \mathrm{yr}^{-1}$ from pure LLR observations. Compared to measurements based on radar observations (Pitjeva 2005), the LLR results seem to be less accurate by a factor of about 8 .

The Nordtvedt parameter has been improved by a factor of 1.5 and agrees with the value derived by Williams et al. (2009). This is again mainly a result of the longer data series and to a smaller extent the refined modelling. At the epochs of possible maximum amplitude, there are either no (new Moon) or fewer (full Moon) observations because of the too high background noise during the measurements. This means that, at the moment, the full potential of the EP test with LLR is not being exploited. New measurements, e.g., by the APOLLO station (Murphy et al. 2008), at full Moon phases will certainly lead to further improvement. In the parametrized post-Newtonian (PPN) framework, $\eta$ is related to the PPN parameter $\beta$ and $\gamma$ by $\eta=4 \beta-\gamma-3$. This equation is valid under the assumption of no compositioninduced EP violation and no preferred frame effects. By taking $\eta$ from this paper and the parameter $\gamma$ derived from the Cassini mission (Bertotti et al. 2003), the PPN parameter $\beta$ has been determined to be $\beta-1=(0.3 \pm 1.3) \times 10^{-4}$.

Both relativistic values discussed in this paper do not show any significant deviation from their Einsteinian values $\left(\dot{G} / G_{0}=0, \eta=0\right)$ and confirm the upper limit to a possible violation of general relativity.

\section{Conclusions}

Owing to the long data series of observations, LLR has become a powerful tool for determining relativistic parameters with high accuracy. Here, the variations in the gravitational constant and the Nordtvedt parameter have been investigated by applying an analysis model that had been extended by incorporating the fluid lunar core influence in combination with an updated data series up to December 2009. We obtained $\dot{G} / G_{0}=$ $(-0.7 \pm 3.8) \times 10^{-13} \mathrm{yr}^{-1}$ and $\eta=(-0.6 \pm 5.2) \times 10^{-4}$. The results of these parameters have been improved by a factor of about 2 relative to previous IfE results, and confirm or even slightly improve the results of other analysis groups for an upper limit to a possible violation of general relativity.

Acknowledgements. Current LLR data are collected, archived and distributed under the auspices of the International Laser Ranging Service (ILRS). We acknowledge with thanks, that the more than 40 years of used LLR data has been obtained under the efforts of the personnel at the Observatoire de la Côte d'Azur in France (9067 NPs used in this analysis), the LURE Observatory in Maui, Hawaii (402 NPs), the McDonald Observatory in Texas (6551 NPs) as well as the Apache Point Observatory in New Mexico (600 NPs). We would also like to thank the International Space Science Institute (ISSI) in Bern for supporting this research.

This research was funded by the Centre for Quantum Engineering and SpaceTime Research QUEST and the DFG, the German Research Foundation, within the research unit FOR584 "Earth rotation and global dynamic processes".

\section{References}

Bertotti, B., Iess, L., \& Tortora, P. 2003, Nature, 425, 374

Biskupek, L., \& Müller, J. 2009, in Proceedings of the Journées 2008 Systèmes de référence spatio-temporels, ed. M. Soffel, \& N. Capitaine, LohrmannObservatorium and Observatoire de Paris, 182-185, les Journées Systèmes de référence spatio-temporels and X. Lohrmann-Kolloquium, Dresden, Germany, 22-24.09.2008

Biskupek, L., Müller, J., \& Hofmann, F. 2009, in Proc. of IAG 2009 Scientific Assembly Geodesy for Planet Earth, International Association of Geodesy Symposia (Springer), accepted

Damour, T., Piazza, F., \& Veneziano, G. 2002, Phys. Rev. Lett., 89, 081601 Hinderer, J., Legros, H., \& Amalvict, M. 1982, Geophys. J. Int., 71, 303

McCarthy, D. D., \& Petit, G. (ed.) 2004, IERS Conventions (2003), (Verlag des Bundesamtes für Kartographie und Geodäsie)

Müller, J., \& Biskupek, L. 2007, Classical and Quantum Gravity, 24, 4533 Müller, J., \& Nordtvedt, K. 1998, Phys. Rev. D, 58, 062001

Müller, J., Williams, J. G., \& Turyshev, S. G. 2008, in Lasers, Clocks and DragFree Control: Exploration of Relativistic Gravity in Space, ed. H. Dittus,

C. Lämmerzahl, \& S. G. Turyshev, Astrophysics and Space Science Library, 349,457

Murphy, T. W., Adelberger, E. G., Battat, J. B. R., et al. 2008, PASP, 120, 20

Nordtvedt, K. 1968a, Phys. Rev., 169, 1014

Nordtvedt, K. 1968b, Phys. Rev., 169, 1017

Nordtvedt, K. 1995, Icarus, 114, 51

Nordtvedt, K. L., Müller, J., \& Soffel, M. 1995, A\&A, 293, L73

Pitjeva, E. V. 2005, Astron. Lett., 31, 340

Sanders, A. J., Gillies, G. T., \& Schmutzer, E. 2010, [arXiv: 1005. 3226]

Soffel, M., Klioner, S., Müller, J., \& Biskupek, L. 2008, Phys. Rev. D, 78, 024033

Steinhardt, P. J., \& Wesley, D. 2010, [arXiv: 1003.2815]

Turyshev, S. G. 2008, Ann. Rev. Nucl. Part. Sci., 58, 207

Williams, J. G., \& Boggs, D. H. 2009, in Proc. of the 16th Int. Workshop on Laser Ranging, 13-17.08.2008, Poznań, Poland, ed. S. Schillak, 1, 101

Williams, J. G., \& Folkner, W. M. 2009, IAU Symp. 261, BAAS, 41, 882

Williams, J. G., Boggs, D. H., Yoder, C. F., Ratcliff, J. T., \& Dickey, J. O. 2001, J. Geophys. Res., 106, 27933

Williams, J. G., Turyshev, S. G., \& Boggs, D. H. 2009, Int. J. Mod. Phys. D, 18, 1129

Zerhouni, W., \& Capitaine, N. 2009, A\&A, 507, 1687 